\title{
EMBEDDING OF ORLIZ-SOBOLEV SPACES IN HÖLDER SPACES
}

\author{
Vesa Lappalainen and Ari Lehtonen
}

\section{Introduction}

For a smooth domain $\Omega$ in $\mathbf{R}^{n}$, e.g. a bounded Lipschitz domain, each function $u$ which belongs to the Sobolev space $W^{1, p}(\Omega)$ is in fact Hölder-continuous in $\bar{\Omega}$ if $p$ is greater than $n$ (cf. Adams [1], Kufner et al [6] or Necăs [14]). A similar embedding property holds also for Orlicz-Sobolev spaces (cf. [1] or [6]).

Typically, the boundary behaviour of $u$ is handled by straightening the boundary to a half space using local coordinate maps and deriving estimates for the Hölder norm of $u$ in terms of the (Orlicz-) Sobolev norm (cf. [14, Chapter 2.3.5.]). Instead of using estimates on the boundary we first show that if $p>n$ the Sobolev spaces $W^{1, p}(\Omega)$ can be embedded in a certain local Hölder class $\operatorname{loc} \operatorname{Lip}_{\alpha}(\Omega)$, $\alpha=1-n / p$ for any domain $\Omega$. The embedding to $C^{\alpha}(\bar{\Omega})$ is then derived for a large class of domains via the embedding of $\operatorname{loc}_{\operatorname{Lip}}(\Omega)$ to $C^{\alpha}(\bar{\Omega})$. The following result is obtained as a corollary:

Theorem. If $\Omega$ is a bounded uniform domain and $p>n$, then $W^{1, p}(\Omega)$ is continuously embedded in $C^{\alpha}(\bar{\Omega})$.

Note that by a result of $\mathrm{P}$. Jones [5] there exists an extension operator $W^{1, p}(\Omega)$ $\rightarrow W^{1, p}\left(\mathbf{R}^{n}\right)$ for uniform domains, and the theorem hence follows from the wellknown embedding $W^{1, p}\left(\mathbf{R}^{n}\right) \rightarrow C^{\alpha}\left(\mathbf{R}^{n}\right)$. However, for the theorem no extension result is needed, and our approach is based on classical Hölder continuity estimates together with Gehring and Martio's [3] and Lappalainen's [7] results on $\operatorname{Lip}_{h}{ }^{-}$ extension domains. Therefore our method applies to a larger class of domains than uniform domains.

\section{Preliminaries}

An Orlicz function is any continuous map $A: \mathbf{R} \rightarrow \mathbf{R}$ which is strictly increasing, even, convex and satisfies

$$
\lim _{\xi \rightarrow 0} A(\xi) \xi^{-1}=0, \quad \lim _{\xi \rightarrow \infty} A(\xi) \xi^{-1}=\infty .
$$

We let $\Omega$ denote a domain in $\mathbf{R}^{n}$. The Orlicz class $K_{A}(\Omega)$ is the set of all measurable functions $u$ such that

$$
\int_{\Omega} A(u(x)) d x<\infty
$$


and the Orlicz space $L_{A}(\Omega)$ is the linear hull of $K_{A}(\Omega)$. As norm in the Orlicz space we use the Luxemburg norm

$$
\|u\|_{A, \Omega}:=\inf \left\{r>0: \int_{\Omega} A(u(x) / r) d x \leq 1\right\} .
$$

The Orlicz-Sobolev space $W^{1} L_{A}(\Omega)$ is the set of functions $u$ such that $u$ and its first order distributional derivatives lie in $L_{A}(\Omega)$. In the case where $A(\xi)=\xi^{p}$ we obtain the standard Sobolev space $W^{1, p}(\Omega)$. For a more detailed discussion of Orlicz spaces we refer to [1] and [6].

A domain $\Omega$ in $\mathbf{R}^{n}$ is called $c$-uniform if each pair of points $x, y \in \Omega$ can be joined by a rectifiable curve $\gamma$ in $\Omega$ such that $l(\gamma) \leq c|x-y|$ and

$$
\operatorname{dist}(\gamma(t), \partial \Omega) \geq c^{-1} \min (t, l(\gamma)-t) .
$$

A modulus of continuity is any concave positive increasing function $h:[0, \infty[$ $\rightarrow \mathbf{R}, h(0)=0$. A function $u: \Omega \rightarrow \mathbf{R}$ belongs to the local Lipschitz class $\operatorname{loc}_{\operatorname{Lip}_{h}}(\Omega)$ if there exist constants $\left.b \in\right] 0,1\left[\right.$ and $M=m_{b}$ such that for each $x \in \Omega$ and $y \in B_{b}(x):=B(x, b \operatorname{dist}(x, \partial \Omega))$

$$
|u(x)-u(y)| \leq M h(x, y) ;
$$

here and hereafter $h(x, y):=h(|x-y|)$. As a matter of fact, it is shown in [7] that it is equivalent to require the condition to hold for $b=1 / 2$; the smallest $m_{1 / 2}$ defines a seminorm of $u$. It should be remarked that this definition differs from the standard definitions of local Hölder spaces. In fact, the class $\operatorname{loc}_{\operatorname{Lip}}(\Omega)$ is not a local space but semiglobal in a sense. A function $u$ belongs to the Lipschitz class $\operatorname{Lip}_{h}(\Omega)$ if there exists a constant $M \leq \infty$ such that $(2.1)$ holds for all $x, y \in \Omega$. For bounded domains $\operatorname{Lip}_{h}(\Omega)=C^{h}(\bar{\Omega})$, where $C^{h}(\bar{\Omega})$ is as in [1, 8.37].

Let $h$ and $g$ be two moduli of continuity. A domain $\Omega$ is a $\operatorname{Lip}_{h, g}$-extension domain if $\operatorname{loc}_{\operatorname{Lip}}(\Omega)$ is continuously embedded in $\operatorname{Lip}_{g}(\Omega)$. For short $\operatorname{Lip}_{h, h}=$ : $\operatorname{Lip}_{h}$ and, for $h(t)=t^{\alpha}, \operatorname{Lip}_{h}=: \operatorname{Lip}_{\alpha}$. The following result due to McShane [13] justifies the name extension domain (see also Stein [15], [3] and [7]).

2.1. Theorem. If $\Omega$ is a $\operatorname{Lip}_{h}$-extension domain and $u \in \operatorname{loc} \operatorname{Lip}_{h}(\Omega)$, there exists a $\operatorname{Lip}_{h}$-extension $u^{*}: \mathbf{R}^{n} \rightarrow \mathbf{R}$.

We can characterize $\operatorname{Lip}_{h}$-extension domains by using the following metric in $\Omega$ :

$$
h_{\Omega}(x, y):=\inf _{\gamma(x, y)} \int_{\gamma} \frac{h(\operatorname{dist}(z, \partial \Omega))}{\operatorname{dist}(z, \partial \Omega)} d s(z),
$$

where the infimum is taken over all rectifiable curves $\gamma$ in $\Omega$ joining $x$ to $y$. 
2.2. Theorem. A domain $\Omega \subset \mathbf{R}^{n}$ is a $\operatorname{Lip}_{h, g}$-extension domain if and only if there is a constant $1 \leq K(\Omega, h, g)<\infty$ such that

$$
h_{\Omega}(x, y) \leq K g(x, y)
$$

holds in $\Omega$.

For a proof see e.g. [3] or [7].

\section{Embedding of Orlicz-Sobolev spaces}

Let $A$ denote an Orlicz function. If

$$
h(t):=\int_{t^{-n}}^{\infty} \frac{A^{-1}(r)}{r^{1+1 / n}} d r
$$

is finite at $t=\varepsilon$, then $h$ defines a modulus of continuity on the interval $[0, \varepsilon]$. It is easily seen that the derivative $h^{\prime}(t)=n A^{-1}\left(t^{-n}\right)$ is decreasing.

3.1. Proposition. If $h(1)<\infty$, then $W^{1} L_{A}(\Omega)$ is continuously embedded in $\operatorname{loc} \operatorname{Lip}_{h}(\Omega)$ for any domain $\Omega \subset \mathbf{R}^{n}$.

Proof. It follows from [1, Theorem 5.35] applied to balls contained in $\Omega$ that each function $u \in W^{1} L_{A}(\Omega)$ is continuous. Now let $B_{b}\left(x_{0}\right)$ be a ball contained in $\Omega$ and $x_{1} \in B_{b}\left(x_{0}\right)$. Let $t:=\left|x_{0}-x_{1}\right|$ and choose a ball $B$ of radius $t$ such that $x_{0}, x_{1} \in B \subset B_{b}\left(x_{0}\right)$. We denote by $|B|$ the Lebesgue measure of $B$ and by

$$
u_{B}:=\frac{1}{|B|} \int_{B} u(z) d z
$$

the mean value of $u$ in $B$. As in [1] we obtain the following estimate for $x \in B$ :

$$
\left|u(x)-u_{B}\right| \leq \frac{2 t}{|B|} \int_{0}^{1} r^{-n} \int_{B_{r}}|\nabla u(z)| d z,
$$

where $B_{r}$ denotes a ball of radius $r t$ contained in $B$. Since

$$
\int_{B_{r}}|\nabla u(y)| d y \leq 2 r^{n}|B|\|\nabla u\|_{A, B_{r}} A^{-1}\left(r^{-n} /|B|\right),
$$

we obtain

$$
\left|u(x)-u_{B}\right| \leq \frac{4}{n \Omega_{n}^{1 / n}}\|\nabla u\|_{A, \Omega} \int_{1 /|B|}^{\infty} \frac{A^{-1}(r)}{r^{1+1 / n}} d r,
$$

where $\Omega_{n}:=|B(0,1)|$. Since $h$ is increasing and concave, we have $h(s t) \leq$ $h((1+s) t) \leq(1+s) h(t)$ for $s, t>0$, and therefore

$$
\begin{aligned}
\left|u\left(x_{0}\right)-u\left(x_{1}\right)\right| & \leq \frac{8}{n \Omega_{n}^{1 / n}}\|\nabla u\|_{A, \Omega} h\left(t \Omega_{n}^{1 / n}\right) \\
& \leq \frac{8\left(1+\Omega_{n}^{1 / n}\right)}{n \Omega_{n}^{1 / n}}\|\nabla u\|_{A, \Omega} h\left(x_{0}, x_{1}\right),
\end{aligned}
$$

which yields the desired result. $\square$

The following theorem is an immediate consequence of Proposition 3.1. 
3.2. Theorem. Let $A$ be an Orlicz function and $h$ defined by (3.1). Assume $h(1)<\infty, g$ to be a modulus of continuity and $\Omega$ to be a $\operatorname{Lip}_{h, g}$-extension domain. Then $W^{1} L_{A}(\Omega)$ is continuously embedded in $\operatorname{Lip}_{g}(\Omega)$.

However, $\operatorname{Lip}_{h, g}$-extension domains do not necessarily exist. In order to apply Theorem 3.2 we need to know that they do exist.

3.3. Theorem. Let $h$ be a modulus of continuity. Then the following conditions are equivalent:

(1) There are constants $K<\infty$ and $t_{K}>0$ such that for every $0<t \leq t_{K}$

$$
\int_{0}^{t} \frac{h(s)}{s} d s \leq K h(t) .
$$

(2) All bounded uniform domains are $\operatorname{Lip}_{h}$-extension domains.

(3) The unit ball in $\mathbf{R}^{n}$ is a $\mathrm{Lip}_{h}$-extension domain.

(4) There exists at least one $\operatorname{Lip}_{h}$-extension domain.

For a proof see [7, p. 27].

Note that if Condition 3.3.(1) holds for all $t>0$, then all uniform domains are $\operatorname{Lip}_{h}$-extension domains.

3.4. Corollary. Assume $A$ to be an Orlicz function with

$$
\frac{A^{\prime}(\xi)}{A(\xi)} \geq \frac{p}{\xi} \quad \text { for a.e. } \xi \geq \xi_{0} .
$$

for some $p>n$ and $\xi_{0}>0$ and $\Omega$ to be a bounded uniform domain.

Then $W^{1} L_{A}(\Omega)$ is continuously embedded in $C^{h}(\bar{\Omega})$, where $h$ is defined by (3.1).

Proof. We just combine Theorem 3.2 with $g:=h$ and Theorem 3.3 with the following lemma.

3.5. Lemma. Let $t_{K}:=A\left(\xi_{0}\right)^{-1 / n}$ and $K:=p /(p-n)$. Then, for $0<t \leq$ $t_{K}, h(t)$ is finite and

$$
\int_{0}^{t} \frac{h(s)}{s} d s \leq K h(t)
$$

Proof. Integrating the inequality (3.2) we obtain $A(\xi) \geq\left(A(\eta) / \eta^{p}\right) \xi^{p}$ for $\xi \geq \eta \geq \xi_{0}$ by the absolute continuity of $A$, and hence $A^{-1}(r) \leq\left(\eta A(\eta)^{-1 / p}\right) r^{1 / p}$ for $r \geq A(\eta)$. Now for $\eta=A^{-1}\left(t^{-n}\right)$ the definition (3.1) of $h$ yields

$$
h(t) \leq \frac{\eta}{A(\eta)^{1 / p}} \int_{t^{-n}}^{\infty} \frac{r^{1 / p}}{r^{1+1 / n}} d r=\frac{\eta}{A(\eta)^{1 / p}} K n t^{1-n / p} .
$$

Since $h^{\prime}(t)=n A^{-1}\left(t^{-n}\right)$ and $\eta / A(\eta)^{1 / p}=A^{-1}\left(t^{-n}\right) t^{n / p}$, we have $h(t) \leq K h^{\prime}(t) t$. 


\section{Examples}

Let $\Omega$ be a bounded uniform domain in $\mathbf{R}^{n}$.

4.1. Let $p>n$ and $\alpha:=1-n / p$. Then $W^{1, p}(\Omega)$ is continuously embedded in $C^{\alpha}(\bar{\Omega})$. This follows immediately from Corollary 3.4 since for $A(\xi):=\xi^{p}$ we have $A^{\prime}(\xi) / A(\xi)=p / \xi$.

4.2. Let $A(\xi):=e^{\xi}$. Then the modulus of continuity defined by (3.1) is given by $h(t)=n^{2}(\ln (1 / t)+1) t$. For any $\left.\alpha \in\right] 0,1\left[\right.$ the Orlicz-Sobolev space $W^{1} L_{A}(\Omega)$ is compactly embedded in $C^{\alpha}(\bar{\Omega})$. By Corollary $3.4, W^{1} L_{A}(\Omega)$ is continuously embedded in $C^{h}(\bar{\Omega})$. Since $h(t) / t^{\alpha} \rightarrow 0$ as $t \rightarrow 0$, the result follows from the Ascoli-Arzela theorem.

4.3. Let $A(\xi):=\xi^{n}(\ln (\xi))^{q}$ and assume $q>n$. Then $h(t)=n(\ln (\eta))^{-q / n} \times$ $(n /(q-n) \ln (\eta)+1)$, where $\eta:=A^{-1}\left(t^{-n}\right)$. Then, if $\Omega$ has the strong local Lipschitz property in $\mathbf{R}^{n}$, the Orlicz-Sobolev space $W^{1} L_{A}(\Omega)$ is continuously embedded in $C^{h}(\bar{\Omega})$. This follows from [1, Theorem 8.36]. However, the modulus of continuity $h$ does not satisfy Condition 3.3.(1) and therefore there does not exist any $\operatorname{Lip}_{h}$-extension domains.

The snowflake or the Koch curve described in Mandelbrot [10, p. 42] bounds a uniform domain whose boundary is very irregular. Examples of domains which are $\operatorname{Lip}_{\alpha}$-extension domains but not uniform can be found in [7] and [3]. Also, in [7] there are examples of $\operatorname{Lip}_{\beta}$-extension domains which are not $\operatorname{Lip}_{\alpha}$-extension domains for any $\alpha<\beta$. 


\section{References}

[1] Adams, R.A.: Sobolev spaces. - Pure and Applied Mathematics 65. Academic Press, New York-San Francisco-London, 1975.

[2] Gehring, F.W., and O. Martio: Quasidisks and the Hardy-Littlewood property. Complex Variables Theory Appl. 2, 1983, 67-78.

[3] Gehring, F.W., and O. Martio: Lipschitz classes and quasiconformal mappings. - Ann. Acad. Sci. Fenn. Ser. A I Math. 10, 1985, 203-219.

[4] Gehring, F.W., and B.S. Osgood: Uniform domains and the quasihyperbolic metric. J. Analyse Math. 36, 1979, 50-74.

[5] Jones, P.: Quasiconformal mappings and extendability of functions in Sobolev spaces. Acta Math. 147, 1981, 71-88.

[6] Kufner, A., O. John and S. Fučik: Function spaces. - Noordhoff International Publishing Leyden; Academia, Prague, 1977.

[7] Lappalainen, V.: Lip $_{h}$-extension domains. - Ann. Acad. Sci. Fenn. Ser. A I Math. Dissertationes $56,1985$.

[8] Lappalainen, V.: Local and global Lipschitz classes. - Seminar on Deformations, LódźLublin. To appear.

[9] Lehtonen, A.: Embedding of Sobolev spaces into Lipschitz spaces. - Seminar on Deformations, Lódź-Lublin. To appear.

[10] Mandelbrot, B.: The fractal geometry of nature. - W.H. Freeman and Company, San Francisco, 1982.

[11] Martio, O.: Definitions for uniform domains. - Ann. Acad. Sci. Fenn. Ser. A I Math. 5, 1980, 179-205.

[12] Martio, O., and J. Sarvas: Injectivity theorems in plane and space. - Ann. Acad. Sci. Fenn. Ser. A I Math. 4, 1978/79, 383-401.

[13] McShane, E.J.: Extensions of range of functions. - Bull. Amer. Math. Soc. 40, 1934, $837-842$.

[14] NeČAS, J.: Les méthodes directes en théorie des équations elliptiques. - Masson et $\mathrm{C}^{\text {ie }}$ Editeurs, Paris; Academia, Editeurs, Prague, 1967.

[15] Stein, E.M.: Singular integrals and differentiability properties of functions. - Princeton University Press, Princeton, New Jersey, 1970.

University of Jyväskylä

Department of Mathematics

Seminaarinkatu 15

SF-40100 Jyväskylä

Finland

Received 7 October 1987 